\title{
El Toque de Midas
}

\section{The Midas Touch}

\section{Eugenio MatiJaseVIC • Bogotá, D.C.}

Cuando llegué a Acta Médica Colombiana en 1988 como corrector de pruebas, los trabajos de investigación que se recibían para evaluación y posible publicación venían escritos a máquina, los correctores corregían directamente sobre los originales, a los autores se les devolvían por correo terrestre las galeradas para su aprobación, el editor revisaba las artes finales en cianotipo (blueprint) antes de dar su imprimatur, la revista se imprimía en Offset, por ninguna parte se veía un computador en las oficinas de Acta Médica y la World Wide Web (la "red") era apenas un sueño. El primer día el Editor General se reunió conmigo y con mis tres colegas de corrección de pruebas y declaró cuál sería nuestra responsabilidad: garantizar que en cada número publicado, en cada artículo, no hubiese un sólo error gramatical, ni un sólo error ortográfico, ni un sólo dato en unidades de medida diferentes a las del Sistema Internacional de Unidades y ni una sola contravención a los Requisitos Uniformes del Grupo de Vancouver (1). Él, como editor general, junto con el comité editorial de la revista, se encargarían de garantizar que los artículos publicados fueran sólidos desde el punto de vista científico y, sobre todo, que bajo la fachada de una investigación clínica no apareciera publicada, disfrazada de ciencia, la reseña publicitaria de un producto farmacéutico. En la actualidad, 22 años después, la mayoría de las técnicas de impresión y tecnologías editoriales que encontré al llegar a la revista han sido arrasadas por nuevas técnicas, dispositivos y tecnologías apenas imaginables en esa época (escribo este editorial con el procesador de texto de mi computador, los trabajos remitidos para evaluación y posible publicación, escritos también mediante procesadores de texto, son enviados en medios digitales ópticos o magnéticos o a través de la red, las correcciones se realizan en un procesador de texto, el editor revisa las "artes finales" en un formato de documento portátil -PDF- antes de dar el imprimatur y la revista se imprime mediante un sistema de impresión digital), pero sigo convencido, como lo estaba Fernando Chalem, el Editor General de entonces, de que no es posible abdicar en lo concerniente a los delineamientos fundacionales de la revista.

Y no me refiero solamente a la calidad editorial, a nuestro compromiso con quienes nos leen de entregarles una revista libre de erratas y acorde con las pautas que acogimos al signar los Requisitos Uniformes; me refiero a nuestro compromiso con los autores, con los lectores, con la comunidad científica en general y con la sociedad, de entregar una revista independiente y veraz: una revista científica no es otra cosa que un vehículo de información científica y la información contenida en la revista debe tener tales cualidades que quien la reciba esté seguro de que puede tomar dicha información por cierta y puede aplicar las recomendaciones dadas sin temor a que detrás de dichas recomendaciones exista una motivación oculta o un propósito no explícito (2). Este énfasis de Acta Médica Colombiana en los aspectos no formales de la revista no es exclusivo de nuestra revista, en la actualidad todas las revistas médicas tienden hacia allá. De hecho, el propio Grupo de Vancouver terminó, en este lapso de 22 años, por convertirse en Comité Internacional de Editores de Revistas Médicas (ICMJE: International Committee of Medical Journal Editors) al tiempo que, de manera gradual, extendió su normatividad con respecto a los trabajos enviados a las revistas para evaluación y posible publicación más allá de los aspectos puramente formales (cómo hacer un resumen estructurado, cómo citar las referencias bibliográficas, cómo numerar las tablas, etc.) hasta los aspectos relacionados con los principios éticos de la investigación y de la publicación en revistas biomédicas (3).

En circunstancias sociopolíticas coyunturales y bajo condiciones muy específicas, instancias gubernamentales y organizaciones gremiales de profesionales de la salud (los
Acta Med Colomb 2010; 35: 37-39.

Dr. Eugenio Matijasevic Arcila: Editor General Revista Acta Médica Colombiana E-mail: eugeniomatijasevic@gmail.com Recibido: 30/VI/10 Aceptado: 01/VII/10 
ejemplos son innumerables en la historia de las publicaciones biomédicas) han intentado e incluso logrado influir en el contenido de las revistas (4), pero en general, la mayor amenaza a la independencia de una revista proviene de los sesgos comerciales que los autores de los artículos a publicar puedan tener $(5,6)$. Este tipo de sesgo en la publicación científica se denomina, desde mucho tiempo ha, conflicto de intereses. La definición ya clásica en lo que respecta a las publicaciones biomédicas es la que dieran en 1993 Marcia Angell y Jerome Kassirer, en la actualidad editores eméritos de The New England Journal of Medicine: conflicto de interés es el conjunto de condiciones en las cuales el juicio concerniente a un interés primario tiende a ser influido de manera inapropiada por un interés secundario (7).

Son muchas las voces pesimistas o acomodaticias que afirman que muy probablemente la verdadera independencia es inalcanzable. Es posible que tengan razón: en un mundo globalizado en el que los intereses comerciales permean prácticamente cada actividad humana, perseguir una pretendida pureza en la que las publicaciones científicas sean ajenas a la realidad mundana parece un sueño utópico, pero una cosa es aceptar que el mundo está hecho así y otra muy distinta permitir que ese hecho pase desapercibido. En realidad no estamos en contra de la presencia de intereses comerciales en el mundo científico, pero sí estamos en contra de que dichos intereses permanezcan ocultos. El verdadero mal está en el disfraz, en que se presente como verdad científica lo que no es más que estrategia de mercadeo y contra ese mal no tenemos otra defensa que la transparencia. Aunque no es posible evitar los conflictos de interés, sí es necesario hacerlos explícitos y, para el caso, la única manera de hacerlo es la declaración de los conflictos de interés. Por ello los Requisitos Uniformes del ICMJE establecen que esta información debe formar parte del manuscrito enviado a evaluación y que se debe incluir en una página aparte inmediatamente después del título. Dicha información debe ser enviada, junto con el trabajo de investigación, a los pares encargados de arbitrar el artículo. Tanto ellos como los futuros lectores deben saber qué lazos del autor o autores con compañías comerciales o con instituciones gubernamentales o con organizaciones gremiales, religiosas o de cualquier otra naturaleza podrían comprometer de una manera u otra la independencia de la investigación. El lector, cualquiera sea el caso, tiene derecho a la información indispensable para juzgar por sí mismo qué tanta credibilidad le otorga a un trabajo de investigación, a una opinión editorial o a una revisión o actualización de temas en los que el autor o autores declaran conflictos de interés.

En general, se ha dicho muy poco con respecto a si estas mismas preocupaciones se deben aplicar a los editores y a los propietarios de las revistas biomédicas, pero en Acta Médica Colombiana consideramos que, en aras de la transparencia, debe ser así y no de otra manera, en la medida en que los conflictos de interés, sean estos de origen ideológico o de origen financiero, también afectan el buen juicio de propie- tarios y de editores. No otra cosa sucede con suplementos famosos en revistas de primera línea que han sido pagados en su totalidad por casas farmacéuticas, en los que desaparece por completo el papel del Editor de la revista e incluso la revisión por pares, y el suplemento es publicado con en el mismo formato de la revista llevando al lector al engaño de considerar que los trabajos publicados en el suplemento en cuestión han recibido el mismo tratamiento y han sido sometidos a las mismas consideraciones que se toman en la elección de un trabajo científico digno de publicarse en la consabida revista (8). En algunos casos puntuales se ha ido incluso más lejos, creando revistas aparentemente arbitradas por pares, con nombres tan pomposos como The Australasian Journal of Bone and Joint Medicine, que, en realidad, son compilaciones de artículos pagados por una compañía farmacéutica interesada en publicitar algunos de sus productos. A una tramoya como estas se prestaron tanto los supuestos editores de la supuesta revista, como la casa editorial (9) sobre quienes recae exclusivamente la responsabilidad moral, ya que la casa farmacéutica que financió el "proyecto" lo colocó bajo el rubro de publicidad y mercadeo $\mathrm{y}$, aparentemente, nadie le puede exigir que no haga publicidad disfrazada de ciencia. En el mundo actual todo cuanto es tocado por el dedo de Midas de la actividad comercial se convierte en oro con el objeto de que sea una inversión que regrese a su vez convertida en el oro de réditos y ganancias para los accionistas. Se trata de una actividad humana en ningún modo ilegítima, pero que, como en la antigua leyenda griega, puede llevar a la consunción: de la misma manera que Midas no podía beber ni comer porque cada bocado que llevaba a su boca se transformaba en oro nada nutricio, la investigación científica no puede ser transformada en oro publicitario sin que deje de ser ciencia.

Sea entonces el momento de hacer una declaración de conflictos de interés con respecto a Acta Médica Colombiana y a su Editor General. Acta Médica Colombiana es el órgano científico oficial de la Asociación Colombiana de Medicina Interna, su propietaria, y es una revista científica en circulación desde 1976, arbitrada por pares, dedicada a la promoción de la investigación científica nacional e internacional en todas las especialidades de la Medicina Interna. Acta Médica tiene un dueño, la Asociación Colombiana de Medicina Interna, Asociación que ha honrado siempre el principio de independencia y libertad editorial adoptado por la Asociación Mundial de Editores Médicos (WAME: World Association of Medical Editors) en el sentido de que el Editor General de una revista es quien tiene autoridad total con respecto al contenido editorial de la revista y a los periodos de estudio y publicación de los trabajos enviados para publicación, sin que los dueños interfieran en la evaluación, selección o edición de dichos trabajos (10).

Puesto que el Editor General es el responsable directo del control de los trabajos de investigación, de los comentarios y de los editoriales que se publiquen en la revista, cualquier conflicto de interés que tenga daría lugar a modificaciones 
sustanciales e inaceptables en el contenido de la misma. Los editores, por tanto, debemos ceñirnos a códigos de conducta éticos y hacer siempre las declaraciones de conflictos de interés que sean pertinentes de acuerdo con el Código de Conducta (Code of Conduct) y a las Guías para la Mejor Práctica de Editores de Revistas (Best Practice Guidelines for Journal Editors) del Comité de Ética en Publicaciones (COPE: Committee on Publication Ethics) (11). En este sentido quiero declarar que no tengo ningún vínculo comercial con ninguna empresa comercial relacionada con la salud, ni poseo acciones de tales compañías, ni recibo honorarios por conferencias dictadas para tales compañías, ni participo en ningún comité asesor de tales compañías y tampoco recibo auxilios, becas, viajes o regalos de ellas.

\section{Referencias}

1. International Steering Committee. Uniform requirements for manuscripts submitted to biomedical journals. Ann Intern Med 1979;90:95-9.

2. Collier J. The price of independence. BMJ 2006; 332: 1447-1449.

3. International Committee of Medical Journal Editors. Uniform Requirements For
Manuscripts Submitted to Biomedical Journals [Internet]. Philadelphia: ICMJE; 2008 [cited 2010 May 30]. Available from: http://www.icmje.org/journals.html

4. Lexchin J, Light DW. Commercial influence and the content of medical journals. BMJ 2006; 332: 1444-1447

5. Smith R. Medical journals and pharmaceutical companies: uneasy bedfellows. BMJ 2003; 326: 1202-1205.

6. Smith R. Medical journals are an extension of the marketing arm of pharmaceutical companies. PLoS Med 2005;2:e138.

7. Kassirer JP, Angell M. Financial conflicts of interest in biomedical research. N Engl J Med 1993; 329: 570-571.

8. Rochon PA, Gurwitz JH, Cheung CM, Hayes JA, Chalmers TC. Evaluating the quality of articles published in journal supplements compared with the quality of those published in the parent journal. JAMA. 1994; 272: 108-113.

9. Elsevier. Statement From Michael Hansen, CEO Of Elsevier's Health Sciences Division, Regarding Australia Based Sponsored Journal Practices Between 2000 And 2005 [Internet]. Philadelphia: Elsevier; 2009 [cited 2010 May 30]. Available from: http://www.elsevier.com/wps/find/authored_newsitem.cws_home/ companynews05_01203

10. World Association of Medical Editors. The Relationship Between Journal Editors-in-Chief and Owners (formerly titled Editorial Independence) [Internet]. Toronto: WAME; 2009 [cited 2010 May 30]. Available from: http://www.wame. org/resources/policies\#independence

11. Committee on Publication Ethics. Code of Conduct and Best Practice Guidelines for Journal Editors [Internet].London: COPE; 2008 [cited 2010 May 30]. Available from: http://publicationethics.org/guidelines 